\title{
Filigrane
}

Écoutes psychothérapiques

\section{L’avenir de la régression dans les traitements psychanalytiques}

\section{Bernard Chervet}

Volume 17, numéro 2, automne 2008

L’avenir du clinicien II

URI : https://id.erudit.org/iderudit/019418ar

DOI : https://doi.org/10.7202/019418ar

Aller au sommaire du numéro

Éditeur(s)

Revue Santé mentale au Québec

ISSN

1192-1412 (imprimé)

1911-4656 (numérique)

Découvrir la revue

Citer cet article

Chervet, B. (2008). L’avenir de la régression dans les traitements

psychanalytiques. Filigrane, 17(2), 41-59. https://doi.org/10.7202/019418ar

\section{Résumé de l'article}

La psychanalyse s'est saisie de l'utilisation très ancienne de la régression à des fins thérapeutiques. La tentative d'améliorer les protocoles de soins ne peut faire l'impasse sur la régression en tant qu'outil. Une étude de cette notion amène à revisiter l'histoire de sa conceptualisation ainsi que l'évolution concomitante de son utilisation dans les cures psychanalytiques. Du point de vue des visées thérapeutiques, une valeur particulière est à reconnaître à la régression d'incidence. D'autres modalités de régressions sont aussi observables, de jour comme de nuit. Du fait de leur proximité avec celle spécifique des protocoles thérapeutiques, elles tendent à prendre sa place et ainsi à devenir des résistances à la guérison. 


\title{
L'avenir de la régression dans les traitements psychanalytiques
}

\author{
bernard chervet
}

\begin{abstract}
La psychanalyse s'est saisie de l'utilisation très ancienne de la régression à des fins thérapeutiques. La tentative d'améliorer les protocoles de soins ne peut faire l'impasse sur la régression en tant qu'outil. Une étude de cette notion amène à revisiter l'histoire de sa conceptualisation ainsi que l'évolution concomitante de son utilisation dans les cures psychanalytiques. Du point de vue des visées thérapeutiques, une valeur particulière est à reconnaître à la régression d'incidence. D'autres modalités de régressions sont aussi observables, de jour comme de nuit. Du fait de leur proximité avec celle spécifique des protocoles thérapeutiques, elles tendent à prendre sa place et ainsi à devenir des résistances à la guérison.
\end{abstract}

$\mathrm{T}$

oute visée prospective est déterminée par notre aperception du passé, par ce que ce dernier dessine comme perspective d'avenir du fait de la puissance de la compulsion de répétition qu'il recèle. Celle-ci peut même s'exprimer par une dramatisation conjuratoire de la compulsion de réduction qui s'y évoque. Le pire est alors toujours à venir.

Une autre source de cette détermination est notre souhait de prémonition issue de nos rêves, en l'occurrence l'espoir que l'avenir soit favorable à la réalisation de nos désirs. Le futur se présente alors à leur image, sous des traits idéalement paradisiaques.

La prise en compte de l'un seul de ces deux déterminants se reflète dans la classique bipartition entre les optimistes et les pessimistes.

Dans le texte qui suit nous allons essayer de garder à l'esprit ces deux réalités de la vie psychique et envisager comment elles participent et tentent ensemble de construire un avenir, certes toujours incertain, à nos patients et à notre discipline ; ceci afin de ne pas nous satisfaire de deux opinions souvent émises : celle affirmant que les traitements psychanalytiques n'ont aucun avenir et celle soutenant que la biologie est l'avenir de la psychanalyse.

La régression banale du fonctionnement mental permet les activités psychiques quotidiennes que sont tant le rêve nocturne que la rêverie diurne. Le premier s'avère indispensable à la santé mentale et participe à l'équilibre somatopsychique; la seconde est partie constituante du tri-pôle diurne actif, passif, réfléchi. La dynamique évolutive existant entre ces trois pôles est elle-même favorable à l'oscillation des fonctionnements diurnes et nocturnes. La régression est donc engagée dans le fonctionnement psychique ordinaire au sein duquel elle participe à la régénération libidinale, à la restauration du narcissisme et à empêcher que l'objectalité ne devienne une voie d'entrée dans la maladie. 
Très tôt la psychanalyse va tenter d'utiliser ces qualités de la régression au service des buts thérapeutiques. La régression va devenir un outil permettant de redonner de l'avenir aux patients qu'elle traite. En tant qu'outil, elle n'est pas un but en soi, mais bien un moyen, le chemin par lequel un sujet pourra récupérer une économie psychique et un certain degré de liberté quant à son utilisation; donc une potentialité d'avenir, de futur authentique.

Le texte qui suit cherche à explorer l'utilisation qui est faite de la régression à des fins thérapeutiques dans les traitements psychanalytiques. Après quelques réflexions sur notre recherche bien légitime de protocoles de soins aptes à obtenir les meilleurs résultats, est étudiée la notion même de régression, l'histoire de sa conceptualisation ainsi que l'évolution concomitante de son utilisation dans les cures psychanalytiques.

Ce parcours permet de bien saisir la valeur de la régression d'incidence du point de vue thérapeutique. D'autres modalités de régressions sont aussi abordées, telles qu'elles apparaissent dans diverses activités psychiques régressives. Par la régression, ces dernières se retrouvent articulées aux protocoles thérapeutiques. Elles en sont des formes adjacentes. Mais elles peuvent aussi, au cours des traitements psychanalytiques, être utilisées transgressivement par les résistances à la guérison et prendre la place de la régression d'incidence, spécifique à ceux-ci.

\section{Méthode de soins et manques processuels}

Les protocoles et méthodes de soins que nous produisons et proposons à nos patients sont bâtis à la lumière de notre appréhension plus ou moins intuitive des achoppements et des faillites de leurs procès psychiques. Plus ces défaillances sont importantes plus les solutions envisagées sont censées apporter une complémentarité apte à contrer les nécessités internes envers lesquelles les patients sont en désarroi. Nos méthodes répondent donc à ce qui, en eux, est dépourvu, et sont, de ce point de vue, des émanations d'un contre-transfert maternel secourable eu égard aux manques processuels.

Ainsi nos techniques de soins sont-elles totalement déterminées par ces manques ainsi que par notre propre rapport au manque; ce qui explique qu'une autre source de nos techniques est le contre-transfert par ressemblance, généralement imbriquée à la précédente, celle liée au contre-transfert par complémentarité. L'identification hystérique se combine alors à l'identification processuelle. Mais nous espérons aussi, ou du moins devraient-ils en être ainsi, qu'en s'offrant comme étayage, nos méthodes et par elles nos propres procès mentaux permettront l'abandon des défenses plus ou moins drastiques, des recours anti-détresse que ces faillites processuelles ont contraint les patients à confectionner.

La psychanalyse participe de cette même logique. Elle est née tout particulièrement des défauts du procès d'endeuillement propre aux hystériques, et aux névrosés en général. Mais elle est née aussi des avatars d'un autre procès, régressif eu égard à celui engagé dans le travail de symptôme, le procès du travail de rêve. La construction du procès d'endeuillement exige en effet le détour par un autre 
procès plus régressif, celui de déformation propre au travail de rêve. Une règle technique se dessine là: l'efficience d'un procès participant à la progrédience ne peut être achevée qu'après un temps de travail préliminaire portant sur un autre procès, régressif, impliqué sur la voie régrédiente. Cette règle suit la logique en deux temps du fonctionnement psychique, celle dite de l'après-coup.

En fait ce sont l'oniromancie et les clefs des songes qui sont les héritières directes des avatars du travail de rêve. Ces techniques refoulantes se doivent de fournir un mode d'interprétation qui vient renforcer le travail de rêve défaillant, ce travail de déformation et de dissimulation, en apportant justement un surcroît de refoulement. La psychanalyse a repris à son compte cet héritage en introduisant dans la cité moderne un nouvel espace d'accueil et d'oubli des rêves et aussi un nouvel apport d'interprétations. Toutefois l'interprétation psychanalytique n'est pas seulement refoulante comme l'est l'interprétation traditionnelle recourant aux symboles; elle se veut d'abord régrédiente, c'est-à-dire occupée à élaborer langagièrement des souhaits et pensées régressives, ceci afin de libérer les procédures du penser diurne des attractions et captations dont il est l'objet de la part des motions pulsionnelles inconscientes. Ce détour est censé lui-même favoriser la réinstauration de l'endeuillement manquant, car écarté.

Nous notons déjà là que toute régression conjugue une attraction régrédiente et une levée partielle de l'exigence progrédiente; toute production régressive est la résultante de ces deux aspects, et est donc un compromis porteur de ces deux enjeux qui ne sont autres que ceux de la dynamique oedipienne pensée en termes processuels, un meurtre conjugué à une sexualisation. La phobie de la régression, voire les attaques dont elle est fréquemment l'objet via la dévalorisation de la méthode psychanalytique, trouve là son origine, dans ce côtoiement régressioncastration (Chervet B., 1992).

Les avatars régressifs du fonctionnement mental ont donc des effets sur l'organisation même de la cité qui se trouve alors contrainte à se doter de lieux d'accueil et d'interprétation, de lieux de soins s'opposant aux attractions régressives et palliant du dehors aux exigences internes manquantes. La contrainte à construire de tels lieux de soins prolonge, reprend sous une forme inversée, les contraintes actives au sein du travail de rêve, du travail de symptôme, du travail de toute psychopathologie. Ces contraintes sont des réponses à des nécessités pulsionnelles extinctives qui n'ont pu être travaillées, traitées par les divers modes d'activités psychiques, celles régressives en particuliers, manque qui a obligé les patients à recourir, face à l'intensité de leur détresse, à des défenses plus ou moins mobilisables, plus ou moins chroniques. Nos outils de soins ont donc un rapport d'homologie inversée avec les procès psychiques manquant chez nos patients. Ils agissent un renfort, voire un apport du dehors.

De là peut naître un degré d'adéquation secourable et, dans le meilleur des cas aussi un degré de dissymétrie tensionnelle favorable à une élaboration des procès restés jusque-là en souffrance; comme cela se dit pour une lettre qui n'est pas retirée, avec le risque que le retrait soit forclos au-delà d'un certain délai. 
Ce préambule a certes une valeur générale. Il nous confronte toutefois à un conflit fondamental, irréductible, propre à toute initiative et toute initiation de nouveaux protocoles de soins. Il nous rappelle que notre attention envers les manifestations tangibles de nos patients se complète toujours d'une perception implicite envers ce qui manque à leur fonctionnement mental; et que c'est ce manque processuel qui est le plus contraignant et qui détermine le plus nos réponses, beaucoup plus que l'excès d'excitation, qui n'en est qu'une conséquence possible, tout comme, à l'opposé, l'inertie et les dépression et déprivation libidinales.

Cet abord nous permet de souligner certains caractères propres à toute démarche thérapeutique. Elle est mue par une aspiration régressive s'imposant à nous. Elle est donc définissable comme une démarche régrédiente qui doit se tourner vers les activités psychiques régressives sous jacentes à nos paroles et nos actions, activités de pensées dont nombre d'actes et de fonctionnements mentaux sont malheureusement privés et orphelins. Cette contrainte infléchit notre intérêt et notre attention, elle dessine une méthode spécifique à nos métiers, méthode caractérisée par les termes d'écoute régrédiente et de travail régrédient. Une méthode incluant donc la passivité ; une méthode à suivre. Est reconnaissable là la classique attention en égal suspens de la psychanalyse. Que l'on ne s'y trompe toutefois pas, il s'agit d'une méthode thérapeutique faisant du travail sur le régressif un détour, sa visée finale étant de permettre aux patients de vivre avec le monde, de pouvoir profiter des infinies nuances de la gamme de tous les plaisirs et déplaisirs; un détour visant in fine l'objectalité.

Ces propos sur la méthode psychanalytique appellent certes un point de vue comparatif avec les autres méthodes de soins psychiques, qualifiées elles aussi de psychothérapiques, mais non psychanalytiques. En découle aussi la possibilité de repérer quelques points de contact entre la psychiatrie et la psychanalyse par le fait que l'existence de ces deux disciplines est déterminée de façon similaire par les avatars et les achoppements du fonctionnement mental, qu'elles sont censées y répondre et qu'elles en ont la charge du point de vue thérapeutique.

\section{Pluralité et diversité : l'hétérogène et l'incompatible}

Si le fait que les psychiatres et les psychanalystes puissent souhaiter se rencontrer peut paraître aller de soi eu égard à leur objet commun, la vie mentale de leurs patients, l'histoire des fluctuations de ces contacts montre que l'apparente évidence, censée promouvoir des moments féconds, est marquée de dialogues de sourds pouvant atteindre de puissantes querelles qui ne sont pas sans nous étonner. Accordement, complémentarité et convergence sont loin d'organiser les rapports entre ces deux disciplines.

Les aspects de marketing et de mode, dont la définition même est de se démoder (Jean Cocteau), ne sont certes pas à négliger, mais apparaissent, sinon secondaires, insuffisants à produire seuls les puissants facteurs affectifs impliqués dans ces discordes. 
La différence entre le travail du psychiatre et celui du psychanalyste est certainement liée au fait que la psychiatrie a l'avantage et l'inconvénient de ne pas s'être pourvu de conception unique de la vie mentale, à la différence des psychanalystes qui, du fait de leur référence fondamentale à l'œuvre freudienne, pourrait au premier abord apparaître mieux nantis en la matière. La différence traduite en terme de nanti et de dépourvu laisse deviner là un des motifs les plus aptes à fomenter lesdites querelles.

Mais il nous faut encore ajouter que nous avons un autre point en commun lié à notre objet partagé ; celui d'être justement soumis quotidiennement au dépourvu de nos patients, et que ce contact avec le traumatique exige beaucoup de nous, qu'il sollicite certes nos empressements à la réparation, nos esthétisations de la folie, mais aussi nos propensions à fuir nos patients. Ce contact avec le dépourvu a aussi donné lieu à de nombreuses théories étiologiques infantiles, telles celle de la dégénérescence, de la séduction, de l'anti-psychiatrie, du déficit, de la substitution biologique. Cette nécessité anti-traumatique de théoriser une causalité s'accompagne d'un appel à des registres de fonctionnement régressifs, tel celui envisagé ci-dessus, celui de la polémique et de la diatribe consistant à élire quelque ennemi, toujours déclaré plus noir que le précédent.

En effet, la vie mentale est ainsi faite qu'elle ne peut ressentir ou percevoir un manque sans se donner quelque théorie, généralement infantile, théorie causale soutenant plus ou moins implicitement, l'existence d'un monde exempt de toute réalité traumatique. Métapsychologiquement, cette théorisation traduit le travail psychique rendu nécessaire par cette réalité de la castration. Elle réalise en même temps un désir de réussir un déni de ladite réalité. Sur le plan épistémologique, elle produit des théories d'attente. La nature a horreur du vide dit-on, la nature humaine certainement. Le comblement de ce vide, si l'on envisage que ce terme désigne le manque à élaborer un objet perdu, donc une place laissée vide alors qu'elle devrait être occupée par quelque représentation douloureuse, n'épuise toutefois pas la question d'une réalité d'un manque en soi, qu'il soit désigné du terme de néant, d'irreprésentable, de non-chose etc. En psychanalyse, nous disposons du terme de castration, terme qui a l'humour et le paradoxe, aux fins d'atténuation, de dire une condensation. Il renvoie à une théorie causale inconsciente, la castration par le père, théorie exigée par le fait que la castration n'est pas représentable en elle-même; donc une théorie contre un manque de représentation; il renvoie encore à un affect, celui de l'effroi ; à une réalité corporelle, l'absence de pénis sur le bas-ventre féminin; à un ressenti d'angoisse se reconnaissant dans l'entendu de certains messages et dans le vu de certaines perceptions externes; et in fine à un fonctionnement mental organisé en deux temps, le procès de l'aprèscoup faisant que la pensée est bivalente, bidirectionnelle selon les voies régrédiente et progrédiente.

Ainsi la psychiatrie se trouve-t-elle en fait plutôt située à un carrefour de théories qu'à une absence de théories. Nous pourrions même considérer que le principe de la psychiatrie consiste à réaliser un exercice délicat, d'équilibriste, 
celui de se maintenir en suspens d'adoption d'une théorie concernant le fonctionnement mental des patients, position de laquelle aura pourtant à être déduit des techniques de soins. Ce carrefour est donc un carrefour de choix et de heurts. Et comme tout carrefour d'indécidabilité, il peut être investi subrepticement par toutes sortes de théories plus ou moins conscientes, de théories infantiles et de confort.

Les choses se complexifient encore si l'on envisage par ailleurs qu'une multiplicité de points de vue psychanalytiques s'est déployée durant le $\mathrm{xx}^{\mathrm{e}}$ siècle. Certes les conceptions freudiennes ont-elles été ainsi enrichies, mais les travaux se sont distribués selon deux pôles; entre un approfondissement de certains aspects de la théorie de Freud, pouvant être intégrés à sa conception générale, considérée dès lors avoir un point de vue plus large et plus fondamental que tous les apports postérieurs, et un autre pôle assurant au contraire que Freud est dépassé, que son œuvre est à ranger dans le domaine de l'histoire des sciences, voire au musée des idées étranges et des idées bizarres. Les épigones d'un tel point de vue se ressentent heureusement libérés de la tutelle freudienne et autorisés à produire des théorisations, régressives dans la mesure où ils omettent de les confronter sérieusement à la conception et à l'exigence de la métapsychologie freudienne. Toutefois toutes ces théories dissidentes rendent compte d'une part de vérité du psychisme, part qu'il convient de ne pas négliger, et donc d'examiner. Psychanalytiquement, il n'y a pas d'opinions, il y a des psychés qui soutiennent leur réalité du discours qu'elles énoncent.

Une fois cette qualité plurielle de la psychiatrie posée, et une fois faite la remarque portant sur la pluralité des théories au sein même de la psychanalyse, il nous faut reconnaître un autre degré de similitude entre nos deux professions, audelà même de leur objet commun, eu égard à ce débat entre unicité et pluralité.

Le débat se présente donc entre notre souhait d'avoir une référence uniciste et la réalité de la psyché agencée selon la diversité. Cette diversité relève idéalement de l'hétérogénéité des divers processus psychiques ainsi que des oscillations topiques habituelles progrédience-régrédience, telles la nuit-le jour, le labeurl'érotisme, la solitude-le grégaire etc. Mais il existe une autre diversité de fonctionnement qui se superpose à la première et qui est faite de fonctionnements incompatibles les uns avec les autres. Cette question de l'incompatibilité est la véritable opposition à l'hétérogénéité des divers processus occupant la vie psychique ordinaire. Peuvent coexister à l'intérieur de la vie mentale ces deux diversités, par hétérogénéité et par incompatibilité ; diversités qui de plus ne sont pas plurielles par successivité mais par concomitance et qui soulèvent de redoutables difficultés théoriques et techniques puisqu'elles vont induire des réponses thérapeutiques tout aussi incompatibles les unes avec les autres que ces fonctionnements eux-mêmes (Chervet, 2006).

On ne s'adresse en effet pas au moi du patient de la même façon qu'à ses revendications pulsionnelles, qu'à ses systèmes de valeur, et plus encore qu'à ses tendances négativantes les plus térébrantes. Mais surtout on ne s'adresse pas de la 
même façon à un déni chronique de réalité qu'à un achoppement quant à pouvoir intégrer ladite réalité.

L'exemple le plus marquant dans l'histoire de la psychiatrie a été l'introduction des médicaments qui si, au premier abord, semblent s'opposer radicalement à l'instauration de procès psychiques riches pour la vie mentale, n'en ont pas moins permis, selon l'usage qui en est fait, de rendre possible l'accès à la vie mentale de certains patients et de leur permettre d'instaurer, d'améliorer des procès mentaux en lieu et place, plus ou moins partiellement bien sûr, plus ou moins définitivement certes, des médicaments. Il convient donc de bien avoir à l'esprit que les aspects lénifiants, calmants, ou stimulants recherchés par les médicaments sont bel et bien des réponses aux avatars de certains procès psychiques, mais aussi qu'ils sont une fin en soi pour certaines modalités de fonctionnement psychique construites à partir de ces avatars et qui les réclament. L'usage de la chimie n'est plus alors une voie ouverte vers l'instauration des procès en souffrance. Certaines méthodes thérapeutiques s'avèrent en effet complices d'une éradication des processus de pensée; elles tirent l'humain à sa simplification.

Nous pouvons nous référer là aux incompatibilités qui sont actuellement soutenues, au nom de l'efficacité, par les autorités de santé censées promouvoir des méthodes thérapeutiques. Certaines recommandations ne s'encombrent pas de la moindre intelligibilité de la morbidité, de la moindre significativité du visible comme aboutissement d'une complexe processualité qui n'a rien à envier ni à la physiologie, ni à la biologie. Il ne s'agit plus que d'assurer l'invisibilité.

Ces propos nous permettent d'insister sur un point essentiel: nous ne pouvons faire notre travail, aborder la vie mentale d'un autre, sans avoir en nous-mêmes quelque théorie plus ou moins officielle du fonctionnement mental idéal, mais surtout des théories implicites, régressives et inconscientes, que nous agissons à notre insu et qui donnent aux psychiatres et aux psychanalystes leurs profils, et plus encore que leur style, leur idéologie.

Ce référentiel, ce fonctionnement mental idéal, va soutenir une conception de la fonctionnalité de l'appareil psychique, soutenir en fait une téléologie, une finalité et une visée de la matière psychique. Cette référence va donc aussi dessiner une dynamique ayant pour but de réaliser cette téléologie, et aussi une topologie, un agencement d'instances rendu indispensable par les nécessités sous-jacentes mettant en danger à tout moment ce but même de la vie mentale.

La psychanalyse s'est dotée d'une formule et d'un outil exprimant et imposant une telle téléologie, sa règle fondamentale qui soutient la visée du devenir conscient, c'est-à-dire la liaison entre tout matériau régressif et la conscience. La psychiatrie a aussi ses visées, probablement plusieurs, tels que le soulagement, l'accueil, le soin, la sauvegarde, mais aussi parfois l'ordre public, en fait souvent la barrière à la dégradation négativante et à la désinsertion; plus fondamentalement heureusement, l'humanisme. 


\section{Régression, régrédience, régressivité}

Une fois replacée dans ce contexte général, nous pouvons aborder plus strictement cette particularité du fonctionnement psychique dénommée régression, ainsi que les notions qui l'accompagne, celles de régrédience (Botella C. et S., 2001) et de régressivité, sans risquer de les cliver artificiellement du reste du fonctionnement.

Donc, la régression; sa valeur dans le fonctionnement mental et par voie de conséquence la place que nous devons lui accorder au sein des traitements psychiques, en particulier bien sûr dans les tableaux où justement elle semble être contournée, suite à quelques difficultés, au profit de la seule voie progrédiente, ou au profit d'une compulsion de répétition inscrite au sein de tableaux dits régressifs, de tableaux signalant non pas un retour en arrière, mais un arrêt dans le développement psychique, donc une distorsion de celui-ci.

Je vais tout d'abord préciser quelques aspects définissant la régression, ceci en m'étayant sur l'histoire de ce concept. Celui-ci en effet unit et sépare dès le début la psychiatrie, particulièrement la psychiatrie française et la psychanalyse. Nous verrons aussi que l'évolution du concept a aussi déterminé par voie de conséquence celle des méthodes de soin.

\section{De la notion au concept}

La notion de régression est née de l'observation d'une tendance spontanée des patientes hystériques à se remémorer et à répéter des événements anciens, sous hypnose et hors hypnose. Ces patientes étaient l'objet de la préoccupation des psychiatres de la fin du XIX ${ }^{\mathrm{e}}$ siècle en ce qu'elles venaient contredire leurs tentatives de différencier la psychiatrie et la neurologie. C'est en présentant des tableaux cliniques semblables à ceux neurologiques et en introduisant un degré de réversibilité là où justement ces derniers semblaient en être déprivés que l'hystérie venait contredire la différenciation recherchée. La plasticité des conversions et leur capacité à s'emparer par identification des formes alentours n'étaient pas alors reconnues, ni la contagion sur les médecins et l'exploitation qui les amenaient à présenter répétitivement en spectacle ces patientes, réalisant ainsi une scène, équivalente au rêve typique de nudité honteuse, où l'une jouit en exhibant son dépourvu, entourée qu'elle est de Messieurs endimanchés, indifférents et nantis.

Ainsi la régression a-t-elle été repérée à partir du moment où a pu être envisagé un mécanisme spécifiquement hystérique. La régression a été décrite par Breuer et Freud en 1893-1895, comme le mécanisme pathognomonique de l'hystérie. Celles-ci, l'hystérie et la régression, étaient appréhendées alors par le biais d'une conception générale physiologique, celle de la dégénérescence. Un pas de plus fut franchi quand l'origine du trouble hystérique fut réinterrogée, la conception physiologique étant battue en brèche en grande partie du fait de la réversibilité, mais aussi du fait que ces patientes avaient une tendance spontanée à dire et redire, à réactualiser en parole, et non pas seulement en crises de conversion, des évènements du passé qu'elles mettaient en lien avec leurs symptômes, propos donc spontanés s'accompagnant 
d'une conséquence tout aussi spontanée et remarquables, bien qu'éphémère et hautement réversible, la disparition momentanée desdits symptômes.

Cet attrait pour l'origine, pour la cause, donc aussi pour la fin, la finalité et le pourquoi, se trouve particulièrement impliqué dans la naissance de la psychanalyse. L'une des principales différences existant entre les démarches de Breuer et de Freud, perceptible dès les Études sur l'hystérie, puis rappelée par Freud luimême dans tous ses textes dits d'histoire de la psychanalyse, est cette préoccupation pour l'origine des symptômes, pour l'étiologie. De façon plus spécifique encore, c'est la place accordée par Freud dans cette étiologie, à la sexualité puis à la sexualité infantile, donc à un déterminant régressif, qui a abouti à la consommation de la rupture entre les deux chercheurs et amis. La voie de la régression, en fait son refus ou l'arrêt de celle-ci par un accrochage à quelque fonds sécurisant car tangible (la sensorialité perceptive originaire puis le narcissisme primaire absolu pour Freud, les archétypes de Jung, les signifiants de Lacan, les pictogrammes de Piera Aulagnier, le conflit intranarcissique de Mélanie Klein, le féminin pure de Winnicott, l'objet primaire de Balint, la relation d'objet de Fairbairn, la rêverie maternelle détoxicante de Bion, etc.), n'a cessé depuis d'alimenter les querelles, les ruptures et les scissions.

Dès le début, Breuer, dans sa démarche, ferme cette investigation et sa théorisation en fabriquant un postulat, en fait en érigeant un élément clinique au statut de postulat explicatif, l'état second, l'état «hypnoïde». Pour lui cet état est la condition nécessaire pour que certains événements et souvenirs s'avèrent traumatiques, au sens du choc traumatique de Charcot, et donnent lieu à une réaction sous la forme d'un prolongement morbide, d'un symptôme hystérique. Cette conception de Breuer repose sur une totémisation d'une représentation issue d'une perception empirique. Par sa théorie des états hypnoïdes il semble se différencier de la théorie ambiante du XIX ${ }^{\mathrm{e}}$ siècle, celle partagée par la psychiatrie française, et donc par Charcot lui-même, la théorie de la dégénérescence, responsable des dégradations, rétrécissements et dissociations des capacités mentales envisagées à la source de la morbidité; mais en fait sa conception des états hypnoïdes reste implicitement physiologique, biologique. Breuer renonce à la dégénérescence irréversible, mais ne cherche toutefois pas à expliquer l'origine de ces états hypnoïdes. Il évite ainsi de les placer sous la houlette de quelque théorie connue. Il laisse cette question en suspens, mais surtout refuse de s'en préoccuper. Une théorie «privée», implicite, se laisse deviner sous un tel refus devenu postulat. Notons toutefois que ce n'est pas seulement les contenus des découvertes de Freud qui éloignèrent et effrayèrent Breuer, c'est le fait qu'en n'y succombant pas, Freud transmettait une exigence d'élaboration et de travail psychique, de renoncement et de désenchantement envers lesquels la psyché ne fait que renâcler. Tous les éléments élaborés par Freud, du fait même de leur élaboration, contiennent un message, un impératif d'endeuillement.

La totémisation était aussi en jeu dans la production même de la théorie de la dégénérescence. Mais dans celle-ci l'opération de totémisation est précédée d'un 
procès particulier, d'extension par déplacement d'une observation réalisée auprès de patients atteints de tableaux neurologiques et non pas hystériques. Cette étiologie se prolongea jusque dans les travaux de Janet qui, lui également, plaçait l'ensemble de la pathologie hystérique dans un tel contexte originel, de dissociation des fonctions psychiques. Notons encore que cette extension étiologique était aussi à l'œuvre au sein de la neurologie elle-même puisqu'il s'agissait de prêter à tous les syndromes la même origine que celle des tableaux syphilitiques. Cette extension contenait donc déjà une accusation de la sexualité, considérée responsable de tous les maux.

Ces façons d'ériger un élément d'un tableau clinique au statut de cause, ou de refuser de proposer de nouvelles conceptions tenant compte des nouveaux faits d'observation, découlent d'un besoin de poser un verrou envers cet attrait émanant de ladite quête des origines, en fait de cet attrait exercée et mue par la régressivité particulièrement active dans cette quête, du fait qu'elle s'ouvre aussi sur la traditionnelle rencontre du Diable.

Probablement qu'un des premiers mouvements d'indépendance de Freud envers les conceptions de son époque, concerne cette théorie clôturant toute question avant même que celle-ci ne soit officiellement posée. "It begs the question» put écrire Freud en 1914 quand il évoqua ces théories qui posent une telle réponse a priori, un tel postulat originaire. Certes, les apports de Charcot, la possibilité de faire apparaître et disparaître la symptomatologie hystérique sous hypnose, ainsi que le fait de ne pas restreindre l'existence de l'hystérie aux seul genre féminin, puis ceux de Breuer qui observa la possible disparition des symptômes par le recours à la verbalisation des hallucinations et souvenirs apparaissant sous hypnose, enfin ceux de Bernheim à Nancy qui obtint le récit des souvenirs par simple pression suggestive hors hypnose, étaient déjà toutes en décalage avec la fermeture radicale que proposait l'idéologie ambiante de la dégénérescence. La réversibilité vint pourfendre le consensus et révéler la croyance partagée dont cette théorie était investie.

Freud réalise un pas de plus quand il envisage que les états seconds sont des résultats symptomatiques plutôt que la condition de l'apparition du symptôme. Il défait alors la fausse liaison refoulante présente dans la théorisation de Breuer, fausse liaison construite sur une inversion de la cause et de l'effet.

Comme tout novateur, son premier geste est iconoclaste envers la théorie ambiante et consensuelle. Il rouvre le verrou posé sur la pensée par la croyance collective en la dégénérescence héréditaire et congénitale. Cette théorie avait en fait en arrière-fond, des pensées concernant la vie sexuelle, pensées trouvant en la syphilis leur justification objective et leur rationalisation. La syphilis sert alors à dissimuler le complexe de castration des hommes envers le désir féminin; confère l'image d'Épinal dissimulant sous le masque de la beauté féminine les traits d'une séductrice cherchant à attirer les hommes dans le vice de la sexualité dans le but de leur être fatal; une féminité agent du Diable et de la Mort. Beauté et perdition viennent masquer la phobie du désir féminin, les désirs inconscients que recèle cette phobie, le manque à construire un tel désir. 
La dégénérescence, telle que utilisée au XIX ${ }^{\mathrm{e}}$ siècle par la psychiatrie officielle, a donc valeur de théorie sexuelle infantile, individuelle et transgénérationnelle, et de théorie anti-féminité, de réaction virile. Elle porte sur le complexe de castration et la culpabilité qui en est le ressort bien que présentée comme sa conséquence. Cause et conséquence tendent à nouveau à s'inverser. De plus cette culpabilité peut être empruntée, héritée, exhumée; à l'image de celle que l'on retrouve dans la parabole biblique des fils ayant les gencives agacées du fait que leurs pères avaient pu consommer les raisins trop verts. La faute est sexuelle, ceux qui la commettent sont des dégénérés; la damnation pèse sur les générations à venir.

Cette réouverture de Freud s'accompagne évidemment d'une nouvelle conception de sa part, d'abord implicite puis de plus en plus manifeste, des origines et de l'étiologie.

Si l'indécidabilité du commencement, celle dans laquelle Breuer a tenté de se maintenir, peut être envisagée comme un principe fondamental assurant la poursuite de tout processus de théorisation, elle ne peut empêcher la psyché de se fournir des interprétations, d'établir des liaisons et relations entre les perceptions, les sensations, les affects et les représentations. Il existe en effet une nécessité intrapsychique, une contrainte à produire de telles liaisons de toutes sortes, la plupart s'avérant après coup des «fausses liaisons» et des théories d'attente. La construction de ces fausses théories et théories d'attente, tout comme les théories sexuelles infantiles, assurent une fonction psychique, celle de contre-investir l'attraction régressive, tendant à la désorganisation quant elle n'est pas mentalisée.

Et si le principe d'indécidabilité est une exigence favorable à la révision et au dénouement des théories de l'origine, nécessaires tant que celles-ci n'ont pas suffisamment fait leurs preuves, il est en même temps lui aussi une théorie des origines: «Pater incertus, mater certissima». L'attraction négative exige un contre-investissement de ce qui, de la scène primitive, n'est pas représentable, la jouissance des parents, en laquelle l'enfant n'a aucune existence.

Freud va ainsi, lui aussi, proposer successivement un certain nombre de théories, défaisant ses anciennes conceptions au profit de nouvelles tenant compte d'un nombre croissant d'observations empiriques.

Ainsi, la première conception de Freud, de cette attraction régressive par un noyau originaire, nous pouvons la trouver sous sa plume, dans les Études sur l'hystérie, dans le dernier chapitre (chap. IV : Psychothérapie de l'hystérie), écrit par lui seul. Il s'agit de l'existence d'un «noyau pathogène» attracteur, imposant un cheminement à rebours, nommée d'abord par Breuer rétrogradation, rétrogression, puis par Freud régression. Breuer avait en effet, le premier, pu observer, au cours d'une tentative de traitement d'âme, cette propension consistant en un double mouvement de retour à une époque antérieure et de répétition chronologique de cette époque passée. Souvenons-nous de la reviviscence que vécut Anna O. au cours du traitement avec Breuer, des deux années 1881 et 1880, jour après jour, chaque jour répétant successivement le même jour des deux années précédentes. Breuer, de plus, remarque avec perspicacité que cette verbalisation 
chronologique des souvenirs hallucinés se corrèle à un à rebours similaire des symptômes correspondants. Il n'envisage pas l'existence d'une attraction par un souvenir plus spécifique que les autres, autour duquel ceux-ci se seraient organisés.

Freud par contre, dans le chapitre IV, décrit avec précision ce cheminement à rebours. Il repère que la remémoration se fait selon un ordre inversé eu égard à l'apparition des symptômes. Et que le succès, la guérison, n'est obtenu qu'une fois les symptômes les plus anciens résolus. Cet à rebours s'effectue selon différentes modalités de strates, temporelles, associatives et formelles, au sein desquelles les souvenirs se frayent progressivement un tel cheminement régressif vers un «noyau pathogène ». Ce dernier, il envisage alors qu'il a pour contenu pathognomonique d'abord la sexualité, puis la sexualité infantile. Enfin il affirme que ce qui fait la pathogénie, c'est un rapport de séduction précoce caractérisé par un écart, un décalage d'âge, voire de générations, entre un «grand» et un «petit».

Nous savons qu'il lui faudra des années pour réinscrire dans sa théorie de la vie pulsionnelle cette attraction régressive en tant que telle. Il en fera alors une caractéristique de la pulsion elle-même; ceci après avoir montré que le rêve est une formation régressive et avoir reconnu que le narcissisme est aussi une organisation régressive. Les pathologies post-traumatiques l'obligent à reconnaître que l'attraction négativante est propre à la pulsion elle-même, que celle-ci est par nature régressive, qu'elle tend à un retour à un état antérieur, et cela jusqu'à l'inorganique. Le traumatique implique dès lors le pulsionnel même, par la régressivité extinctive.

Chemin faisant, Freud nous propose un certain nombre de conceptions et de théories qui, après-coup, peuvent être considérées comme des théories d'attente. La première fut bien sûr cette théorie de la séduction évènementielle qui, après que Freud ait pu y renoncer, laissera place à une théorie de la séduction intrapsychique qui donne lieu d'abord à la théorie du fantasme pathogène puis à celle des fantasmes originaires, en tant qu'ils représentent les rapports de tension et d'échanges, les articulations inter-instantielles de l'appareil psychique. Ainsi l'attraction du ça sur le moi naissant, le fait que les désirs inconscients tendent à s'emparer d'un moi ainsi séduit par cette attraction, devient la théorie qui remplace et libère la métapsychologie de l'événementialité simple d'une séduction d'un enfant par un adulte, mais aussi de l'accusation du fantasme lui-même en tant que porteur auto-érotique du désir. Il faudra encore des années pour que Freud conjugue à cette attraction régressive pulsionnelle la part revenant dans ce qui fait la dimension traumatique, à l'éradication de l'impératif surmoïque.

Ainsi, la recherche étiologique de Freud, cette préoccupation qui fut à l'origine de la psychanalyse, a été progressivement remplacée par un objet métapsychologique, la conception de Freud de la régression dans laquelle la valeur traumatique du sexuel dépendra du contre-investissement constitué par la désexualisation organisant les soins parentaux. Ainsi ceux-ci ne pourront participer à la mise en place d'une topique intrapsychique de leur enfant qu'à condition qu'ils ne soient 
pas agis, en fait qu'ils ne soient pas transmis sans l'impératif de contre-investissement qui les maintient fantasmes inconscients, mais aussi ainsi en modifie radicalement la nature et les effets.

Nous trouvons là la part de vérité présente dans toutes les théories accusatrices de la sexualité et qui, reprises en morale, se présentent sous la forme d'un complexe de castration, d'une théorie reliant étroitement le désir et la castration, faisant de la seconde la conséquence du premier. Ont donc été d'abord retenue, après les dégénérescences syphilitiques, un mésusage de la sexualité, usage régressif puisque auto-érotique ou marqué par l'abstinence; une sexualité régressive, une sexualité orientée vers le passé, une sexualité infantile, un mésusage de la sexualité fixée aux objets de l'enfance, qualifiable alors d'incestueuse, puis plus précisément une sexualité tournée vers les grandes institutions du moi, vers le narcissisme, mettant l'appareil psychique en danger; mais ce sera seulement tardivement que cette part de vérité sera reconnue comme étant une qualité propre à la pulsion, la qualité primordiale de la pulsion, celle d'être régressive dans son essence même. Un danger apparaît alors lié à la régressivité pulsionnelle; mais ceci à la condition qu'elle soit livrée à elle-même, que l'autre pôle, celui qui retient, organise et oriente la pulsionnalité, qui la contraint à s'inscrire partiellement en contre-investissement narcissique, à renoncer pour une part à ce que sa satisfaction soit de l'ordre de l'extinction, «une fois pour toute», que cet autre pôle ne soit pas éliminé, liquidé, objet d'un «meurtre». Le danger se complexifie alors puisqu'il inclus le rôle de ce qui est constitutif du travail psychique, les opérations impliquées dans les divers procès psychiques, la processualité à strictement parler, celle sous-jacente au déroulement des processus-activités psychiques. Se trouve donc impliqué un principe basal, l'impératif à réaliser les diverses modalités de travail psychique, tant ceux régressifs que ceux progrédients. Ainsi peut-on affirmer que la dimension traumatique strictement psychique est constituée de cette régressivité pulsionnelle et du risque encouru par la processualité, et donc de la tendance à éliminer l'impératif processuel.

Ces propos compliquent particulièrement ce qui peut être appelé régression, et aussi origine psychique, puisque nous venons successivement de trouver et l'attraction extinctive, et l'exigence élaborative, c'est-à-dire l'entrée en scène d'un impératif processuel réclamant l'élaboration et la résolution.

\section{Évolution de la méthode thérapeutique}

Ces aspects d'histoire n'ont pas comme seul intérêt de cerner le déroulement de l'évolution qui a permis à la notion de régression en tant que phénomène descriptif d'accéder au statut de concept métapsychologique. Il trace en même temps une histoire de l'évolution des traitements psychanalytiques. En effet, rappelons l'isomorphie existant entre la tendance dite spontanée des patientes hystériques à suivre une associativité à rebours, à se remémorer, à frayer langagièrement des voies rétrogrades vers un dit noyau pathogène, avec la démarche de Freud occupé qu'il était par sa recherche de l'étiologie de l'hystérie. Cette recherche étiologique 
fut dès l'origine imbriquée à une démarche thérapeutique, et eut pour conséquence immédiate des modifications de celle-ci.

C'est en effet au cours de traitements psychiques que Freud a pu individualiser tout d'abord la régression comme mécanisme fondamental spécifique des névroses, puis comme mécanisme appartenant à l'ensemble du fonctionnement psychique, puis à l'ensemble de la pensée, permettant alors d'aborder et de décrire un grand nombre de fonctionnements psychiques qualifiables d'activités psychiques régressives de la passivité dont le prototype est bien sûr le rêve. Cet élargissement a permis de sortir de la conception d'une régression symptôme pour en faire un mécanisme appartenant à la vie mentale; puis de se rendre compte, de façon quasi inversée eu égard au point de départ, que ces activités psychiques régressives de la passivité participaient tout au contraire à promouvoir la bonne santé psychique. Du premier mouvement thérapeutique consistant à faire sortir les patients de la régression pourra advenir une seconde conception du travail thérapeutique consistant alors à améliorer, instaurer, promouvoir les activités régressives au sein du fonctionnement mental global. Nous sommes passés de la régression-signe pathognomonique de la névrose, donc à traiter, à un mécanisme tout au contraire utile aux fins thérapeutiques et à améliorer lui-même.

Les traitements analytiques visent donc l'instauration du travail régressif de la vie mentale, en même temps qu'ils utilisent la tendance régressive pour aboutir à cette fin. La régression est devenue progressivement l'outil royal de la répétition du passé tant par la remémoration que par la répétition, l'agieren de transfert. Il faudra encore des années pour qu'elle soit comprise comme un moment d'un procès beaucoup plus ample, moment indispensable à la réalisation d'une fonction précise, anti-traumatique, fonction ayant pour but de traiter la nécessité qui traverse la psyché sous la forme de la régressivité extinctive et qui par cette fonction pourra régénérer libidinalement l'ensemble de la psyché.

Chemin faisant ont pu être décrites des méthodes thérapeutiques qui, après coup, s'avèrent être des techniques privilégiant des moments partiels participant tous à un procès de plus grande envergure, celui de l'après-coup. Ce procès d'une rare complexité n'est intelligible que si nous prenons en considération la double polarité constituant le traumatique, déjà désignée plus haut, la régressivité extinctive et l'impératif d'élaboration. Cette fonction de l'après-coup, fonction économique, a pour but de ressourcer la psyché et de porter ainsi à la disposition de la conscience diurne des primes de libido disponibles aux multiples destins des actions volontaires.

Retraçons rapidement ce cheminement en soulignant ce qu'il nous apprend sur la constitution de la voie régrédiente.

Freud a donc successivement connu et pratiqué l'hypnose (Charcot), la méthode cathartique de Breuer, puis la suggestion de Bernheim. Il gardera de ces diverses méthodes la part de vérité qu'elles contiennent, tout en les articulant à une exigence d'élaboration, exigence à la base des modifications qu'il fit subir à ces méthodes jusqu'à la mise en place d'une nouvelle méthode, dite freudienne, nommée par lui psychanalyse. 
En 1895, quand il aborde avec Breuer la régression par la rétrogradation et la remémoration, leur attention se porte vers ce qu'ils vont décrire comme un «blocage» des affects. Il s'agit donc d'obtenir par la réitération, voire même de force, les souvenirs des événements anciens porteurs de ces affects «bloqués», «coincés» et ainsi une catharsis de ceux-ci. Le but thérapeutique repose sur ce déblocage d'affects.

Puis le noyau traumatique s'enrichit d'un contenu précis, sexuel. Il s'agit de retrouver des souvenirs pathogènes sexuels, de l'adolescence mais surtout de la petite enfance. La méthode thérapeutique consiste alors en une élaboration associative, en des retrouvailles d'évènements et l'effacement des effets de ceux-ci; en une perlaboration de ces expériences anciennes dites de séduction précoce.

Puis, à partir de 1900, la régression, retrouvée dans le travail de rêve sous la modalité de la régression formelle et non plus de conversion, se fait pour Freud vers la sensorialité perceptive originaire ${ }^{1}$. Il s'agit de retrouver l'expérience sensorielle originaire avec l'objet, expérience à partir de laquelle sont nées les représentations. Le but du traitement est de reconstituer les traces de l'enfance, de combler l'amnésie infantile, de reconstituer le puzzle de cette amnésie infantile, en particulier le puzzle de la sexualité polymorphe de l'enfant. Les notions de puzzle de l'amnésie, de complétude de la levée de l'amnésie, trouvent là leurs racines et poursuivent une visée d'intégralité.

En 1914, Freud réenvisage la régression mais cette fois dans une conception globale d'un narcissisme primaire absolu attracteur. Le sujet serait dominé par la tentative de retrouver un état narcissique absolu connu originairement au sein de sa mère. Ceci amène, au niveau de la ,technique thérapeutique à porter particulièrement attention sur les conditions favorables au développement mental. La théorisation de Freud se porte sur ces conditions, et sur le couple mère-enfant. Se développent alors la théorie des identifications fondatrices du moi et une technique qui, par le biais du transfert, tente de retrouver, de modifier et de réinstaurer des identifications plus favorables au fonctionnement mental.

Cette position de Freud, même s'il ne la récuse pas, est réouverte par lui très rapidement, deux ans plus tard. En effet, il remet en cause son socle du narcissisme primaire absolu et réintroduit la dimension traumatique comme mise en cause de la capacité d'un système narcissique à se maintenir. Certes, Freud va-t-il hésiter quant à l'origine de ce traumatique, entre la puissance des excitations venant de la réalité extérieure et une tendance intrinsèque aux sources pulsionnelles, à la pulsion elle-même. Il va alors introduire la troisième qualité de la pulsion, sa régressivité, sa tendance au retour à un état antérieur jusqu'à l'inorganique, et à travers ces notions, la pulsion de mort.

Dès lors, le traumatique est à envisager comme endo-pulsionnel, comme la tendance propre à la pulsion à s'éteindre elle-même, et non pas seulement à se décharger mais à empêcher sa constitution même. Cet aspect va être traumatique tant pour Freud que pour tous les psychanalystes puisqu'il n'y aura plus aucun moyen assuré pour se défendre radicalement de la tendance traumatique ; la psyché 
se trouve dès lors condamnée à exécuter un travail psychique, de jour comme de nuit; plus de répit. L'idéalisation de l'être humain en prend encore un coup. Bien sûr, en contre-mouvement de cette âpre réalité, toutes les idéalisations vont venir en renfort.

Toutefois, dès lors, le travail thérapeutique va se centrer sur les procès psychiques, les processus engagés dans les différentes instances, le déroulement de ces différents procès, leurs articulations avec comme référence idéale en arrièrefond la mise en place d'un fonctionnement discontinu en deux temps, celui de l'après-coup. La thérapeutique est devenue processuelle.

Chemin faisant Freud précise la métapsychologie de la régression, c'est-à-dire qu'il aborde celle-ci sous les points de vue topique, dynamique et économique. Sont désormais distinguées au sein même de toute formation régressive les participations respectives des régressions, temporelle, celle connue depuis Breuer; topique, c'est à dire celle engagée dans l'oscillation entre le système sommeil-rêve et le système de pensée diurne, donc l'oscillation système narcissique-système objectal; puis celles conséquentes, libidinale, objectale, etc.

La relecture globale de l'œuvre de Freud, nous permet, riche que nous sommes de la dynamique intégrale de sa théorisation, de percevoir que la conception de la régression de l'interprétation du rêve, envisagée à cette époque comme un retour à l'image sensorielle première, comparée à la conception solipsiste proposée en 1914 d'un narcissisme primaire absolu, a la valeur d'une fixation au représentable. De même ces deux propositions, celle d'une régression au représentable et celle aux conditions d'instauration de la psyché, apparaissent elles-mêmes être des fixations défensives anti-traumatiques, eu égard à celle au masochisme primaire exigée par la régressivité extinctive de la pulsion de mort, telle que abordée par Freud en 1920 et 1924. Ces conceptions constituent donc des solutions symptomatiques, hallucinatoires et d'attente, dissimulées dans des théories scientifiques.

Cela dit, de cette longue évolution, Freud et les psychanalystes après lui, auront à garder la part de vérité de chacune des étapes et à reconnaître ces dernières en leur articulation en le procès d'ensemble qu'est l'après-coup. Ainsi, ce travail sur la processualité psychique ne peut-il se faire sans les contenus de remémoration, sans la répétition nécessaire à l'instauration des identifications, sans la possibilité de réanimer les affects «coincés», et surtout sans la prise en compte finale d'une tendance propre à la psyché à nier elle-même, au nom de toute théorie idéalisante, le fait qu'elle soit occupée par une tendance qualifiable de destructrice, en fait tendance annihilatrice, s'opposant à son existence même. La dimension de réaction thérapeutique négative passe au premier plan du souci thérapeutique, ainsi que le travail sur les procès psychiques et sur les points de fixation régressifs ayant pour but de maintenir déniée l'irréductible réalité de cette opposition à la vie mentale. La castration, affirmée par Freud comme ayant un rôle fondamental très tôt dans son œuvre, devient en effet absolument centrale et se trouve étroitement associée aux procès psychiques qui ont comme fonction de la traiter plutôt que de la reconnaître, traitement qui toutefois aboutit à sa reconnaissance. 
Notons encore que cette évolution de Freud sera reprise par lui-même quand il examinera une logique regroupant et différenciant, du point de vue technique, remémoration et répétition (1914). Il poursuivra cette démarche plus avant en envisageant que toute la psychopathologie peut être placée sous la houlette de sa célèbre formule comme quoi les patients «souffrent de réminiscence» (1895; 1937). Remémorations, répétitions, compulsions et constructions appartiennent donc toutes à la catégorie des réminiscences, doivent faire l'objet d'une investigation, et ainsi servir la visée thérapeutique. Tous les traitements psychanalytiques ont à suivre cette réalité de la réminiscence selon les divers modes par lesquels elle se présente, la remémoration, la répétition, les compulsions, la construction, et tous doivent apprendre à suivre ces procès et à les rendre utiles au fonctionnement psychique général (Chervet, 2006).

\section{Les activités psychiques régressives}

Revenons à la valeur de la régression, donc aux visées de ce travail thérapeutique, de cette construction de la voie régrédiente, de cette mentalisation de la régressivité en activités psychiques régressives ordinaires. Celles-ci exigent toutes un certain degré de passivité, donc une mise en latence plus ou moins importante du pôle actif.

Nous avons déjà souligné que c'est par son étude du rêve que Freud va pouvoir sortir la régression de sa première identité de mécanisme psychopathologique, qu'il va amorcer le schéma d'un fonctionnement psychique idéal incluant le travail particulier de la voie régrédiente et donc rendre possible l'appréhension de ces activités psychiques régressives banales par le biais de la description de l'une de ces occurrences, la régression formelle. Succinctement, celle-ci articule un déni temporaire et réversible de la réalité objectale, une désobjectalisation, une mise en latence d'une partie du pôle actif, le pôle de la secondarisation, et une transformation de l'encodement des pensées verbales en un autre code, celui du rébus fait d'image. Ces images vont avoir plusieurs identités: celle de maintenir un lien avec le code langagier bien sûr, celle d'être des figures de la sensorialité érogène sous-jacente, celle de représentant-représentations de la pulsion, celle de matériau présentable sur l'écran interne de la conscience. Elle participe ainsi aux trois buts du travail de rêve, réaliser hallucinatoirement un désir, maintenir le sommeil et produire un perceptif saturant la conscience et soutenant le déni inaugural, tous trois reflétant la fonction fondamentale du travail de rêve, sa fonction anti-traumatique consistant à régénérer libidinalement l'ensemble de la psyché, à restaurer le narcissisme et à promouvoir une prime de désir, disponible au réveil à l'objectalité.

Cette modalité de régression ne couvre pas toutes celles que nous avons à vivre bien sûr, mais elle offre un modèle pour comprendre les autres. Ainsi en particulier la régression sensorielle, celle que le travail de rêve a pour but de limiter au cours du sommeil afin d'éviter le réveil, et qui a à s'inscrire dans une autre scène qui lui est spécifique, la scène érotique. C'est elle qui est cultivée, par les préliminaires, dans cette autre scène, érotique. 
D'autres modes de régression doivent encore retenir notre attention. Freud a examiné celle engagée dans les symptômes de la vie quotidienne, cette régression de compromis, agie dans nos lapsus, oublis, actes manqués etc. Elle nous ouvre à celle qui a lieu au cours des séances d'analyse, et qui est favorisée par le protocole divan-fauteuil. La libre association de séance, cette parole spécifique des séances d'analyse, peut en effet être appréhendée et décrite comme une régression langagière, une régression d'incidence, une parole d'incidence productrice de double sens (Chervet, 2006).

Est encore possible de décrire une régression animique, celle typique du jeu des enfants au cours desquels l'enfant utilise des matériaux externes en tant que supports de ses représentations inconscientes, ceci afin de construire en lui les procès nécessaires à sa vie psychique. La répétition est alors l'outil même de cette mutation d'une potentialité en efficience.

Ce qui réunit toutes ces activités, c'est leur rapport et leur façon de traiter fort différemment la dimension traumatique liée à la régressivité pulsionnelle et à l'impératif élaboratif. Elles utiliseront à cette fin soit des matériaux mnésiques, représentatifs, soit des conversions corporelles, soit des objets matériels externes ayant en même temps valeur de représentation pour la psyché, soit encore le code langagier, comme dans les séances. Il s'agit dans tous ces cas de régression mentalisée.

C'est par un travail utilisant l'une de ces modalités d'activités régressives, la parole d'incidence, modalité produite artificiellement par la méthode psychanalytique, qu'il est possible d'obtenir la mutation de la régressivité extinctive en une pensée régrédiente constitutive de la voie régrédiente, permettant la confection d'une multitude de productions et d'activités régressives. Cette mutation a aussi des conséquences sur notre rapport au pôle actif, sur les activités qui lui sont propres et qui sont elles aussi prometteuses de satisfactions, autres.

La conjugaison des deux voies est certainement ce qui promeut au mieux la qualité, les nuances des satisfactions auxquelles nous pouvons prétendre, leur diversité, leur subtilité, ainsi que leur imprévisibilité.

bernard chervet

39 , rue professeur florence

69003 lyon

16 , rue jacques callot

75006 paris

bernard@chervet.fr

\section{Note}

1. «La représentation retourne à l'image sensorielle d'où elle est sortie un jour» (Interprétation du rêve). 


\section{Bibliographie}

Botella, C., Botella, S., 2001, Régrédience et figurabilité. Rapport au Congrès de langue française, Paris 2001, Revue française de psychanalyse, $\mathrm{n}^{\circ} 4$.

Chervet, B., 1992, Régression et castration, Revue française de psychanalyse, $\mathrm{n}^{\circ} 4$.

Chervet, B., 2006, L'exercice de la psychanalyse, in A. Green (dir.), B. Chervet (éd.), Unité et diversité des pratiques du psychanalyste, Paris, Presses universitaires de France.

Chervet, B., 2006, La lumière du rêve et la parole d'incidence, in B. Chervet et C. Jean-Strochlic, Rêve et séance, Paris, Presses Universitaires de France, 2007, 7-60.

Chervet, B., 2008, Les réminiscences de l'infantile, in P. Denis, B. Chervet et S. Dreyfus-Asséo, Les avancées de la psychanalyse, Paris, Presses universitaires de France. 